\title{
Transitivity and proportionality in causation
}

\author{
Neil McDonnell ${ }^{1}$
}

Received: 11 June 2016 / Accepted: 28 October 2016 / Published online: 17 November 2016

C The Author(s) 2016. This article is published with open access at Springerlink.com

\begin{abstract}
It is widely assumed that causation is transitive, but putative counterexamples abound. These examples come in three varieties: switching cases, short circuit cases, and what I will call mismatch cases. In this paper I focus on the mismatch variety, which is widely taken to be the easiest to resolve. I will first introduce the cases and the existing strategy for dealing with them, then present a new counterexample which is immune to that strategy. In response to this new counterexample I will introduce a novel solution, one drawing on Yablo's proportionality principle for causation. There is a catch, however. Either proportionality is a strong constraint - it constrains which causal claims are true-and the solution works, or it is not and causation is not transitive after all. I will argue that the first horn has unacceptable consequences and should be rejected, but that the second horn may be less costly than it initially appears.
\end{abstract}

Keywords Transitivity · Proportion - Causation - Counterfactual · Counterpart . Chaining

That causation is, necessarily, a transitive relation on events seems to many a bedrock datum, one of the few indisputable a priori insights that we have into the workings of the concept. (Hall 2000, p. 198)

Is the relation of causation transitive? If $c$ is a cause of $d$, and $d$ is a cause of $e$ does it follow of necessity that $c$ is a cause of $e$ ? To many, the transitivity of causation seems fundamental and yet in recent years several apparent counterexamples, which purport to show failures of transitivity in fairly ordinary cases, have appeared. These putative

Neil McDonnell

neil.mcdonnell@gmail.com

1 Philosophisches Seminar, Universität Hamburg, Von-Melle-Park 6, 10. Stock, 20146 Hamburg, Germany 
counterexamples come in three distinct varieties: switching cases, short circuit cases, and what I will call mismatch cases. In this paper I will introduce a new problem for the existing responses to mismatch cases, before going on to propose a novel solution. I will conclude that causation is not transitive, but, to address the mismatch cases at least, we do not need it to be.

\section{The transitivity thesis}

The transitivity thesis can be stated as follows:

TRANSITIVITY: If $c$ is a cause of $d$ and $d$ is a cause of $e$, then $c$ is a cause of $e$.

According to this thesis when I cause the phone to ring and when that ringing wakes someone up, then it is true that I cause that person to wake up. Since such causal chains are entirely commonplace it is no wonder that causation is widely assumed to be transitive.

However, several putative counterexamples to TRANSITIVITY have emerged in recent years. These examples have three distinct structures. One sort are known as switching cases, where some initial $c$ causes $d 1$ rather than $d 2$ to occur, and where $d 1$ causes $e$, but where the conclusion that $c$ is a cause of $e$ is unpalatable. Here is an example:

\section{Switch:}

A train is proceeding down the main track and a switch is flipped, directing the train temporarily onto a side track. The tracks later converge and the train arrives at the station on schedule, just as it would have had it remained on the main track the whole time.

Here we have a chain of causation from the flipping of the switch $c$ to the train's travelling on the side track $d 1$, and from the journey on the side track $d 1$ to the timely arrival at the station $e$. However, it is specified that either track ( $d 1$ or $d 2)$ would have taken to the station on time ( $e$ ), so all $c$ does is switch how $e$ came to pass, not whether it did. This leads many to think that $c$ should not be considered a cause of $e$, which contradicts TRANSITIVITY.

Another sort, are so-called short circuit cases, in which a threat is created, then cancelled by the same original cause $(c)$ such that the eventual effect, $e$, happens just as it would if $c$ hadn't occurred. Here is an example:

\section{Short Circuit:}

A bomb is placed under the bench where Suzy is sitting. Suzy spots it and calls the bomb-squad who defuse the bomb. Suzy gets a clean bill of health the next day. ${ }^{1}$

Here we have $c$ (placing the bomb) causing some intermediate $d$ (defusing) which cancels the threat $c$ posed. It is also the case that the defusing of the bomb $(d)$ caused

$\overline{1}$ This example is widely attributed to an unpublished work by Hartry Field. 
Suzy's subsequent health $(e)$. Thus, by transitivity the placing of the bomb caused Suzy's good health, which seems like the wrong result.

In both switching and short-circuit cases, there is no question that there is a causal chain running from $c$ to $e$, and yet it is widely taken as false that $c$ causes $e$. I think that there is much to be said about these cases and their status as counterexamples but, whilst I will return to discuss them briefly towards the end, they are simply not the topic of this paper and will be set aside.

Instead I will focus on a third, seemingly simpler, sort of case which I will name mismatch cases. These are cases in which $c$ causes $d$ and where $d$ causes $e$, but where it is clear that the difference $c$ made to $d$ is irrelevant to $d$ 's contribution to $e$. Here is an example:

\section{Purple Flame:}

Jones puts some potassium salts into a hot fire. Because potassium compounds produce a purple flame when heated, the flame changes to a purple colour, though everything else remains the same. The purple flame ignites some flammable material nearby. Here we judge that putting the potassium salts in the fire caused the purple flame, which in turn caused the flammable material to ignite. But it seems implausible to judge that putting the potassium salts in the fire caused the flammable material to ignite. (Menzies 2014) ${ }^{2}$

In this example it seems that we have a case of $c$ being a cause of $d$, and $d$ being a cause of $e$, but intuition dictates that $c$ is not a cause of $e$ : the salts did not cause the flammable material (curtains, say) to ignite. If our intuition about the salts and the curtains is to be trusted then this case also stands as a counterexample to TRANSITIVITY. Only one counterexample is required to refute the thesis, so whatever the verdict in the cases of switching and short-circuits, if this is a genuine counterexample, then TRANSITIVITY is false.

The available responses to this example are: (i) accept that causation is not transitive and survey the damage; (ii) insist that causation is transitive and bite the bullet by accepting the counter-intuitive conclusion that the salts caused the curtains to ignite; (iii) show that there is a problem with the example.

In this paper I will discuss two approaches to (iii). First I will give lay out some background issues concerning the causal relata, then I will argue, as others have, that there is an implicit shift in the middle event $(d)$ which nullifies the Purple Flame example. However, I will further argue that we can create new, harder, examples which are not open to the same response. In Sect. 3 I will turn to providing an alternative approach. I will first introduce Yablo's proportionality constraint, which would rule out Purple Flame and its more difficult successors as genuine counterexamples. In Sect. 4 I will consider some problems for this alternative approach, and offer a novel refinement.

\subsection{Background: the causal relata}

To aid this discussion I will apply a simple counterfactual test for causation. A fullblown counterfactual analysis of causation would be highly controversial to assume in

\footnotetext{
2 This example is originally due to Ehring (1987, p. 323).
} 
such a discussion and so here I commit only to the following far weaker, and much less controversial, causal test: counterfactual dependence between distinct events is prima facie evidence of a causal connection, and the lack of such dependence is prima facie evidence of a lack of causal connection. For simplicity I will be adopting a broadly Lewisian reading of the relevant counterfactuals: $e$ counterfactually depends upon $c$ iff, in all of the closest possible worlds where $c$ does not occur $(\neg c), e$ does not occur $(\neg e) .^{3}$ So, to use a familiar example, it is true on this test that Socrates's drinking hemlock caused him to die because if he had not drunk the hemlock $(\neg c)$ then he would not have died $(\neg e)$. Importantly for Lewis, when we consider what it is for an event not to occur, we cannot simply consider a barely different version of the event (a sip less hemlock, say), but rather a clean excision of the event, 'leaving behind no fragment or approximation of itself' (Lewis 2004, p. 190). ${ }^{4}$

I will also be taking a particular stand on how we should think of the causal relata. The above counterexample to transitivity only has bite if the effect in the first step and the cause in the second are one and the same. If we distinguish the effect of Jones' adding the salts $(p)$, from the purple flame which causes the ignition $(f)$, then we have two causal steps: $c$ causes $p$ and $f$ causes $e$. However, these two steps have no common middle term that would, when adopting TRANSITIVITY, licence the absurd conclusion that $c$ caused $e$. So, if we are taking the putative counterexamples seriously, we must account for the sameness of the middle event in each case.

Since I will be taking the examples seriously, I will be assuming that the event relevant to the effect in the first causal step is the same event as the event which is relevant to the cause in the second. There are at least three alternative treatments of the causal relata that can accommodate this assumption. We could, like Schaffer (2005), be contrastivists about causation and think that the burning of the purple flame and the burning of the flame indeed pick out the same event, but that each description prompts us to consider different alternative events that might have taken place instead. Schaffer's approach requires thinking of causation as a four-place, rather than twoplace, relation. Alternatively, we could follow Paul (2000), and treat the relata of causation as event aspects. On this view the effect in the first step and the cause in the second concern the same event, but just two different aspects of that event. Or we could instead adopt a counterpart-theoretic approach to events: take events to be regions of a given world, but allow that they 'occur' in other worlds in virtue of having counterparts in those worlds. Importantly, which regions of other worlds are counterparts is a contextually sensitive matter: it varies with context and with the mode of representation of the events in question. ${ }^{5}$ So, on this view 'the burning of the purple flame', and 'the burning of the flame', both pick out the same region, and so the same

\footnotetext{
${ }^{3}$ Here I use the simplifying device of 'closest' worlds, which implies that there is some closest world(s). Lewis felt that this Limit Assumption was unmotivated (Lewis 2001, pp. 19-21) and I am inclined to agree, but it simplifies the definition of counterfactual dependence here and is harmless for the purposes of this paper.

${ }^{4}$ See also Lewis (1986, pp. 210-211).

${ }^{5}$ For Lewis's original statement of counterpart theory see his (1968). Lewis's account varied importantly across several iterations as shown in Beebee and MacBride (2014). My contextualist and anti-essentialist application of counterpart theory most closely resembles that found in Lewis (2003).
} 
event, but the different descriptions nevertheless imply different conditions for the occurrence or non-occurrence of that event in other worlds.

All three such approaches have the key features I require: each considers the causal relata to be more fine-grained than events; thus each can account for the sameness of the middle place that is required if we are to take the putative counterexamples seriously - there is a common middle event-without conceding that there is thereby a common causal relatum; and each approach is sensitive to the context and the mode of representation of the event when determining which causal relata are being picked out. I expect any account with these features will suffice for making the case that I do here, and nothing is intended to hang on which is adopted.

That said, the contrastive approach is revisionary in a way that will be unnecessary here: it posits a four-place relation where a two-place relation is what common sense would have us expect. Also, whilst I suspect that there is little of substance to choose between the aspects and counterpart-theoretic approaches, and whilst both fit neatly within a counterfactual treatment of causation, the counterpart-theoretic approach will give me a more flexible notation (which I will put to use in Sect. 3). ${ }^{6}$

To illustrate, let us take it as given that drinking hemlock $(c)$ caused Socrates to die (e). According to the counterfactual test for causation introduced above, drinking hemlock caused Socrates to die: it true that had Socrates not drunk the hemlock $(\neg c)$, he would not have died $(\neg e)$. However the pedant might point out that Socrates must die eventually, and so there is no case where $\neg e$ is true. Of course the pedant is trading here on the implicit cross-world identity conditions for the death event $e$. By the pedant's standard, any death of Socrates will do, but by our ordinary standard only a relevantly similar death will be considered a case of $e$ occurring. We capture this in counterpart-theoretic notation as follows: refer to events by lower-case letters $(c$, $e$ etc.) and the set of counterparts related with those events by subscripts ( $m, n$ etc.). Thus, the pedant is associating set of counterparts $m$ with $e$, so they are asking whether drinking hemlock caused $e_{m}$; the ordinary speaker on the other hand is associating $e$ with a different, more restricted, set of counterparts $n$, and thus are asking about whether drinking hemlock caused $e_{n}$. This notation makes clear the implicit shift that the pedant was trading on: drinking hemlock caused Socrates to die roughly as he did $\left(e_{n}\right)$, but it didn't cause him to be mortal $\left(e_{m}\right)$.

\subsection{Counterpart-sensitive transitivity thesis}

The lesson from the Socrates example is this: specifying the event alone is not sufficient to fix the truth of a causal claim, we must also specify the set of counterparts that is to be applied in that context. This suggests that our initial transitivity thesis was underspecified. Here I refine the thesis to incorporate counterpart-sensitivity. ${ }^{7}$

TRANSITIVITY- C: If $c_{m}$ is a cause of $d_{p}$ and $d_{p}$ is a cause of $e_{n}$, then $c_{m}$ is a cause of $e_{n}$.

\footnotetext{
${ }^{6}$ For a fuller exposition of the counterpart-theoretic approach to events, see McDonnell (2016).

7 Again, it is important to emphasise that the counterpart-theoretic approach is just one way (alongside the contrastive and aspects approaches) to bring out this important, implicit, variable. I do not intend anything of substance to hinge on adopting it in this discussion.
} 
This refined transitivity thesis requires that the middle event of the causal chain be associated with the same set of counterparts when it is the effect event in the first step and when it is the cause event in the second. This is a stricter test than the original version, which left the cross-world identity conditions implicit, and thus open to ambiguity. In the next section, I will discuss the putative counterexamples to the transitivity of causation and show how we can address these examples using this stricter version of the thesis.

\section{Putative counterexamples}

I now turn to consider how this refined transitivity thesis fares when faced with certain well-known counterexamples to the traditional transitivity thesis. I will then introduce a novel counterexample which shows that a new strategy is required. I will introduce just such a new strategy in Sect. 3.

Considering Purple Flame: in all of the closest worlds where the potassium salts are not added there remains a flame, just not a purple one. So, 'if there had been no salts then there would have been no flame' is false, but 'if there had been no salts there would have been no purple in the flame' is true. So, the potassium salts caused there to be purple in the flame, but not for there to have been a flame simpliciter.

The removal of the flame would avert the ignition, but simply altering the colour of the flame would not. So, 'if there had been no flame, there would have been no ignition' is true, whereas 'if there had been no purple in the flame, there would have been no ignition' is false.

Conjoining the two true causal claims you get: the salts caused the purple in the flame but the flame simpliciter caused the ignition. There is one middle event but it is being associated with two different sets of counterparts: in the first counterfactual the event is represented as essentially purple whereas in the second it is represented as essentially a flame, and only accidentally a purple one. So, this case does not have the format $c_{m}$ caused $d_{p}$ and $d_{p}$ caused $e_{n}$, but rather $c_{m}$ caused $d_{p}$ and $d_{f}$ caused $e_{n}$. If we remained insensitive to shifts in which counterparts are associated with the events, as the original transitivity thesis does, then this would count as a case of transitivity. However, since the set of counterparts associated with the middle event shifts between the first claim and the second, and since we are adopting the counterpart-sensitive version of the transitivity thesis, this chain is not a candidate for transitivity and so cannot act as a counterexample to the refined transitivity thesis.

Here is another putative counterexample to TRANSITIVITY- $\mathrm{C}$, that shares the mismatch structure:

\section{Dog Bite:}

Terrorist, who is right-handed, must push a detonator button at noon to set off a bomb. Shortly before noon, he is bitten by a dog on his right hand. Unable to use his right hand, he pushes the detonator with his left hand at noon. The bomb duly explodes. (Hitchcock 2001, p. 277) ${ }^{8}$

\footnotetext{
8 This example is attributable to McDermott (1995).
} 
In this case we assume that if the dog bite had not occurred, the button would still have been pressed, just not with the left hand. So, 'if there had been no dog bite then there would have been no press' is false, but 'if there had been no dog bite there would have been no left-handed press' is true. Thus the dog bite is a cause of the left-handed press but not a cause of the press simpliciter.

If there had been no press, the bomb would not have exploded, so it is true that 'the press caused the explosion'. We also know that 'if there had been no left-handed press, then there would have been no explosion' is false, given the set-up of the case. On the assumption that these counterfactuals reveal the causal story, the press simpliciter is a cause of the explosion but the left-handed press is not.

Conjoining the two true causal claims you get: the dog bite caused the left-handed press, and the press simpliciter caused the explosion. There is one middle event but it is being associated with two different sets of counterparts: in the first conjunct the event is represented as essentially left-handed, but only accidentally a pressing whereas in the second it is represented as essentially a pressing and as only accidentally lefthanded. So, this case does not have the format $c_{m}$ caused $d_{p}$ and $d_{p}$ caused $e_{n}$, but rather $c_{m}$ caused $d_{p}$ and $d_{f}$ caused $e_{n}$. If we remained insensitive to shifts in which counterparts are associated with the events, as the original transitivity thesis does, then this would count as a case of transitivity. However, since the set of counterparts associated with the middle place shifts between the first claim and the second, and since we are adopting the counterpart-sensitive version of the transitivity thesis, this chain is not a candidate for transitivity and so cannot act as a counterexample to the revised transitivity thesis.

So, we can freely admit that the Purple Flame and Dog Bite examples do yield absurd results, but the foregoing discussion demonstrates that they do not qualify as examples of transitivity. Not, at least, on the reading of the counterfactuals that I offered. It is essential to this outcome that the set of counterparts associated with the middle event shifts between the first and second causal claims. In Dog Bite, the pressing event is first represented as essentially left-handed, then as only accidentally so, and in Purple Flame the middle event must first be essentially purple, then only accidentally so. A shift in the represented essence of an event yields a shift in the counterparts associated with that event, and it is my contention that the solution to the mismatch cases given above trades on a particular reading of which essence is represented. In the next section, I will raise a new problem for that solution.

\subsection{Dis-ambiguating the middle place}

Discussing transitivity, Mackie (1980) points out that it is a 'very old form of fallacy' to offer 'a syllogism with an ambiguous middle term' and in recent times Paul (2000) and Schaffer (2005) have exploited the strategy of disambiguating the middle term just as I have here. As discussed above, the general strategy accepts that there is a common event involved in each step (in line with common sense), but that this alone does not ensure that there is a common causal relatum. To determine which contrast pair, which event aspect, or which counterpart relation applies, we must attend to the context and the mode of representation. Thus, the general strategy is a contextualist one, and it 
diagnoses an ambiguity in the middle place that the right reading the context can help us resolve.

In the Socrates example discussed earlier, there was just such an ambiguity in the specification of the effect-Socrates's death-that the pedant exploited. In that case, we had to attend to the context to work out how the implicit variable (that determines which counterparts were associated with the event) ought to be filled in. Once the implicit variable is given an explicit value, there is no longer room for such pedantry: Socrates's drinking hemlock caused him to die in roughly that way, and roughly at that time. When we make some information about the event's modality part of the causal claim, we narrow the scope for ambiguity. This helps us fix upon the causal relatum that we are making a claim about: not any old death, but one sufficiently like the actual one. The lesson here is that we can disambiguate the middle term in the transitivity cases above by explicitly delimiting its essence.

I think that this feature of the contextualist strategy can be exploited to create a new, more resilient, counter-example, however. Consider the following example:

\section{Explicit Purple Flame:}

The potassium caused the purple flame (which was essentially purple and essentially a flame), and the purple flame (still essentially both purple and a flame) caused the ignition. ${ }^{9}$

It is important to point out that counterpart theory does not rule out there being a context in which such a rich essence for the purple flame is appropriate-without a further commitment to fixed essences, any set of counterparts can constitute a viable counterpart relation. Similarly, the aspect theorist cannot plausibly rule out doubleaspect causes in general. The iron needs to be hot and heavy to get the creases out, and a bullet needs to be both travelling quickly and made of a rigid material, to pierce the armour. In both cases two aspects of the event are required for the effect.

For ease of reference I will use $d_{p f}$ to represent the burning of the purple flame as essentially both purple and a flame (or as having the double-aspect of being both purple and a flame). If the potassium salts had not been added, then the flame would have been orange and so the flame would not have had both of the essential features/aspects specified in Explicit Purple Flame. Thus the potassium salts $\left(c_{m}\right)$ caused the purple flame $\left(d_{p f}\right)$ on this reading. Similarly, if the purple flame been cleanly excised $\left(\neg d_{p f}\right)$, then there would have been no ignition $\left(\neg e_{m}\right)$, so the purple flame caused the ignition on this reading too. ${ }^{10}$

I think that the new case does formally qualify as an example of transitivity because $c_{m}$ caused $d_{p f}$ and $d_{p f}$ caused $e_{n}$. So, as long as the conclusion that the potassium caused the ignition is absurd, this qualifies as a counterexample to TRANSITIVITY- C. (I omit the equivalent explicit formulation for Dog Bite for brevity.)

\footnotetext{
9 Note, I use "essentially" here as a shorthand for whatever feature or features that every counterpart has. Since the set of counterparts associated with an event can vary across contexts, so too can an event's essence.

10 Here it is important to recall that the cause event is to be cleanly excised and not just replaced by a close alternative, as discussed in Sect. 1.1 above. In the case of the event which is both essentially purple, and essentially a flame, we are to consider the closest worlds where whatever occupies the relevant region is neither purple nor a flame.
} 
It is worth offering some defence of this example, however, as it is crucial to what follows. This case leaves the mechanics of the original Menzies example intact: nothing about the physical region is changed by the specification of the essence, and so nothing about the causal structure should be thought to have changed. All that has been changed is the essence imputed to the event in the middle place. The problem in the original cases of Purple Flame and Dog Bark was that the initial cause was irrelevant to the eventual effect, and that remains true in the new example. What contextualist approaches try to do is distinguish the modality of the middle event $(d)$ in the first clause, with the same event in the second. On a friendly reading of the context, this approach does the job: there are two candidate readings of the middle event in each example which, once disambiguated, highlight a plausible equivocation. Explicit Purple Flame is designed to shine a light on the work being done by the friendly reading of the context that is required for this solution. By showing that an unfriendly context, in this case one in which the event modality is stipulated, yields an unpalatable answer, the contextualist must say what is wrong with the unfriendly reading, or accept that they have not yet solved the original problem. Such a context may be contrived, or unnatural but it is a possible context nevertheless.

What seems to be going wrong in all three cases, and perhaps most clearly in Explicit Purple Flame, is that there is irrelevant detail in the middle place. A simple counterfactual test does not have the resources to defend against such irrelevancy, however: the truth of the relevant counterfactual only establishes that the non-occurrence of one event $(c)$ is sufficient for the non-occurrence of the other $(e)$, not that every part of $c$ is relevant to the occurrence of $e$. For example, if Suzy's throw is a cause of the window breaking on the counterfactual account, it may well be the case that some broader event including Suzy's throw and some irrelevant additional region, or aspects, counts as a cause too. So, if irrelevancy is the issue, then we need an amendment to the counterfactual approach that delivers relevance. It is to just such a proposed amendment that I now turn.

\section{A proportionality constraint}

In the last section I showed that a promising solution to the mismatch counterexamples to TRANSITIVITY- $C$ runs aground when faced with a new, more difficult, sort of case. Whilst such cases are viable as counterexamples, it is clear that they trade on the irrelevancy that a simple counterfactual account allows. In this section I consider a new strategy and key to this strategy is the notion of proportionality. In the next section I will critically assess this approach, and offer a modified alternative.

Suppose that Derek has a triangle that is scarlet and that he places it in front of Sophie, a pigeon that is trained to peck at all and only red things. Sophie then pecks the triangle. What caused Sophie to peck? Consider this causal scenario under two different descriptions:

1. The placing of the red triangle caused Sophie to peck.

2. The placing of the scarlet triangle caused Sophie to peck.

In the first description it seems as though the redness of the triangle is essential and so in any close world in which the placing of the red triangle does not occur, Sophie will not 
peck. Thus the simple counterfactual test I have adopted counts the placing of the red triangle as a cause of Sophie's peck and this seems like the right, intuition-matching, result.

Compare this with the second description which represents the scarletness as essential. In the closest worlds in which the event of placing the scarlet patch is cleanly excised, Sophie will not peck. Hence, the simple counterfactual analysis would count the two causal claims above on a par: both are causes.

The difference between scarlet and red in this sort of case was discussed by Yablo (1992). Yablo argues that the relationship of scarlet to red is that of determinate to determinable where the determinate, $\mathrm{P}$, determines the determinable $\mathrm{Q}$ only if: (i) necessarily, for all $x$, if $x$ has $\mathrm{P}$ then $x$ has $\mathrm{Q}$; and (ii) possibly, for some $x, x$ has $\mathrm{Q}$ but lacks P (1992, p. 252). Roughly, if something is scarlet, it must be red, but if it is red it need not be scarlet. This can be translated into counterpart-theoretic terms: if something is essentially scarlet, then all of its counterparts will also be red, but if it is essentially red then it may well have non-scarlet counterparts.

In the case of Sophie, Yablo points out that citing the determinate scarlet, when citing the determinable red will do, amounts to giving too much information. It need not have been that precise shade to make Sophie peck, so to be that precise about the shade is to be, if not strictly wrong, at least misleading about what was required to make Sophie peck. I may be left thinking, wrongly in this case, that my crimson triangle won't elicit a peck too.

Too little information can be just as bad. Suppose that a second pigeon Alice had been trained to peck all and only scarlet things. Does placing the red triangle cause Alice to peck? If it had not been red, then Alice would not have pecked, so the claim looks true by the lights of the counterfactual test, but intuitively it is much better to cite the scarlet colour of the triangle in explaining Alice's peck. Being too imprecise in respect of the colour of the triangle may mislead: I may be left thinking, wrongly in this case, that my crimson triangle will elicit a peck too.

In the Sophie case, the scarlet was sufficient for the peck, but not required for it-it is not required because any other red would do. In the Alice case the triangle being scarlet is required, but just being red is not sufficient. So, here is a proposal: for a causal claim to be properly formed the cause must be both sufficient and required for the effect. This is the essence of Yablo's proportionality constraint: the cause must be specific enough, but not too specific, with respect to the effect.

More formally, Yablo offers the following definitions:

\section{Proportionality:}

Where $X$ is a fine-grained event defined in terms of some property and where + and - indicate, respectively, more or less specificity or determinateness of the property in question.

sufficient: $X^{-}$is sufficient for effect $E$ iff for every $X^{+}$, if $X^{-}$, had occurred without $X^{+}, E$ would still have occurred.

required: An event $X^{+}$, is required for E iff for every $X^{-}$, if $X^{-}$had occurred without $X^{+}, E$ would not have occurred. 
Yablo's formulation adopts a fine-grained event ontology. As discussed earlier, this fine-graining of events fails to capture the sense in which the purple flame and the flame, the left-handed press and the press, are the same events being described in two different ways. The contrastive, event aspect and counterpart-theoretic accounts of the causal relata were all able to capture the idea that there could be single (coarsegrained) event, but potentially many (fine-grained) causal relata. So, before going on to apply the proportionality principle to the issues concerning transitivity, we must first translate it into one of these alternative conceptions of events, and of the causal relata. Here is where I think the notation we find in the counterpart-theoretic approach is especially helpful (though, again, I do not claim that it is essential).

We can translate the proportionality principle into counterpart theoretic terms by introducing the notion of an event being strictly more fragile or robust when associated with one set of counterparts rather than another. An event $e$, associated with set of counterparts $m$, is strictly more fragile than the same event $e$ when associated with set of counterparts $n$ iff every counterpart of $e_{m}$ is a counterpart of $e_{n}$ and not vice versa. Robustness is just the invert of fragility so $e_{n}$ is strictly more robust than $e_{m}$ iff $e_{m}$ is strictly more fragile than $e_{n}$.

To represent the relative robustness of two sets of counterparts associated with the same event in different contexts, it will help to represent one relation in terms of the other. So, suppose that an event $e$ can be taken to be relatively robust in context $D$. I will refer to its set of counterparts in $D$ as $n$ in that context and write $e_{n}$ when referring to $e$ under counterpart relation $n$. In some other context $C$ in which $e$ is taken to be strictly more fragile than it is associated with set of counterparts $n$, I will refer to that counterpart relation as $c_{n}{ }^{+}$. In some other context $E$ in which $e$ is taken to be strictly less fragile (i.e. more robust) than when it is associated with set of counterparts $n$, I will refer it as $c_{n}{ }^{-}$. This is simply a naming device, like Yablo's $X^{+}$and $X^{-}$(for more and less specific), that helps express the idea that these two counterpart relations have a particular logical relation. On Yablo's account scarlet is a determinate of the determinable red. On my account, the placing the scarlet triangle in front of Sophie is strictly more fragile than the placing of a red triangle in front of Sophie. The subject of the proportionality constraint has moved from properties on Yablo's view, to eventcounterpart pairs on mine. ${ }^{11}$

I think this allows the following analogue of the proportionality constraint, utilising event-counterpart pairs in place of Yablo's properties ${ }^{12}$ :

\section{Proportionality $_{c p}$}

sufficient $_{c p}$ : An event $c$, associated with set of counterparts $m-c_{m}$-is sufficient $c_{c p}$ for effect $e$ iff for every $c_{m}{ }^{+}$, if $c_{m}$ had occurred without $c_{m}{ }^{+}, e$ would still have occurred.

\footnotetext{
${ }^{11}$ It may prove to be an advantage of the counterpart-theoretic approach that it is not restricted to natural properties. If one counterpart relation entails another, but not vice versa, then the first is strictly more fragile than the second regardless of how unnatural or gruesome it is.

${ }^{12}$ I am using Weslake's (2013) paraphrase, and I alter the notation from Yablo's $X^{+}$for more specific and $X^{-}$for less specific to my preferred reference to the robustness or fragility of the event when associated with different sets of counterparts.
} 
required $_{c p}$ : An event $c$, associated with set of counterparts $m-c_{m}$-is required $_{c p}$ for $e$ iff for every $c_{m}{ }^{-}$, if $c_{m}{ }^{-}$, had occurred without $c_{m}$, $e$ would not have occurred.

This way, the placing the red triangle is sufficient ${ }_{c p}$ for Sophie's pecking since had it been crimson, and therefore not scarlet $\left(c_{m}{ }^{+}\right)$but still red $\left(c_{m}\right)$, the triangle would still have made her peck. The redness of the triangle is also required ${ }_{c p}$ for the pecking since if the triangle had been coloured $c_{m}{ }^{-}$, but not red $c_{m}$, the pecking would not have occurred.

The same event, taken as essentially scarlet (i.e. associated with set of counterparts $n$, and noted as $c_{n}$ ), is also sufficient $c_{c p}$ for the pecking since had the triangle been a lighter or darker shade of scarlet $\left(c_{n}{ }^{+}\right)$, Sophie still would have pecked. However the essentially scarlet event $\left(c_{n}\right)$ is not required ${ }_{c p}$ for the pecking since had the triangle still been red $\left(c_{n}{ }^{-}\right)$, but a different shade $\left(\neg c_{n}\right)$ then the pecking would still have occurred.

On this account, the event of placing the triangle is the proportional cause of the pecking when it is the placing of an essentially red triangle, but not when it is the placing of an essentially scarlet triangle. Yablo's suggestion that proportionality is a constraint on a well-formed causal claim would appear to explain the initial, intuitive, reading of the Sophie case: it is preferable to cite the red triangle rather than the scarlet when giving the cause of the peck.

Returning now to the mismatch counterexamples from Sect. 2, it seems that the proportionality constraint lends weight to the interpretation of the events I originally offered. In the first version of the Purple Flame case I took the flame to be essentially purple when it was an effect of the salts and accidentally purple when it was the cause of the blaze. Since the flame simpliciter is sufficient ${ }_{c p}$ and required ${ }_{c p}$ for the blaze, but the purple flame is merely sufficient $t_{c p}$, the proportionality constraint warrants taking the flame simpliciter to be the cause of the ignition, and the purple flame not to be. Since the salts did not cause there to be a flame simpliciter, there is no causal chaining in this case and no counterexample to the transitivity thesis. Only by building too much information into the specification of the middle event did the apparent problem arise.

In the Dog Bite case, the left-handed press is merely sufficient ${ }_{c p}$, for the detonation: since any old press will do, the left-handed press is not required ${ }_{c p}$. Specifying that it was a left-handed press provides too much information and violates the proportionality constraint. So, the bite caused a left-handed press, but a press simpliciter caused the explosion. Since the modality of the left-handed press and the press simpliciter can be distinguished on a counterpart theory of events, there is no causal chain that runs from the bite to the detonation. Once again, the proportionality constraint provides justification for treating the middle place in this putative case of transitivity as having shifted between the first causal step and the second. Where there is no overlapping chain, there is no counterexample to the transitivity thesis.

So, adopting a proportionality criterion lends further support to the contextualist argument that Purple Flame and Dog Bite were not genuine counterexamples because, in my preferred way of speaking, they traded on a conflation with regards to which set of counterparts is associated with the middle event in the chain. However, I offered a harder problem in the case of Explicit Purple Flame. In that harder case the conflation 
is removed by explicitly fixing the set of counterparts which is associated with the event in the middle place so as to ensure a qualifying transitive structure. The harder case was made possible by the need for an interpretive step to establish what set of counterparts should be attributed to the middle event (or which aspect was under consideration) in a given context. The harder case works by overriding the interpretive step:

\section{Explicit Purple Flame:}

The potassium caused the purple flame (which was essentially purple and essentially a flame), and the purple flame (still essentially both purple and a flame) caused the ignition.

In this case there is no room to re-interpret the essences (or aspects) that apply to the middle event because they have been made explicit and, by a counterfactual test, the salts cause the purple flame and the purple flame causes the fire. So, by TRANSITIVITY$\mathrm{C}$ the salts were a cause of the ignition. Unlike the original Purple Flame and Dog Bite cases, this case qualifies as transitive and has an absurd conclusion. It looks like a genuine counterexample.

However, notice how Explicit Purple Flame falls foul of Proportionality ${ }_{c p}$ : the salts are required ${ }_{c p}$ and sufficient $t_{c p}$ for the purple flame but the purple flame is merely sufficient $_{c p}$, not required ${ }_{c p}$, for the ignition since any colour of flame would have done the job of igniting the curtains. The first step is proportional but the second step is not. Once we replace the second step with the proportional claim that the flame simpliciter caused the curtains to ignite, the case is no longer a candidate for transitivity as, once again, the middle place shifts between the first causal claim (where it is essentially purple and essentially a flame) and the second (where it is only accidentally purple). There is no stable position in which the claims are proportional and where TRANSITIVITY- $\mathrm{C}$ would make the salts a cause of the ignition.

So, each mismatch counterexample to TRANSITIVITY- C, when genuinely transitive, fails to meet my revised Proportionality ${ }_{c p}$ constraint. This motivates my central hypothesis in this paper: that chains of causation do not always confer an overall causal connection between the first event and the last, but chains where each step satisfies Proportionality $_{c p}$ (i.e. proportional causal claims) might. In the remaining section, I will consider a dilemma that is raised by this hypothesis.

\section{The dilemma of proportionality}

On introducing Yablo's proportionality constraint I framed it as a constraint on a 'properly formed' causal claim. This was intentionally ambiguous between two readings. On the first, strong, reading it is a constraint on which causal claims can be true. On this reading, an out-of-proportion causal claim is literally false (though it may remain acceptable to say for pragmatic reasons). On the second, weak, reading, the proportionality constraint is not a constraint on which claims are true, but merely a constraint on which causal claims are optimally formed in some sense. On this reading, an outof-proportion causal claim can be true. I think that those in favour of a proportionality 
constraint face something of a dilemma on this point. In this section I will lay out the dilemma, and then give an argument against the strong interpretation of the proportionality constraint. I will then consider what work a weak proportionality constraint can still do in relation to the transitivity of causation.

On the one hand, it seems as though proportionality must be a strong constraint if it is to do the work I have put it to in the forgoing discussion of transitivity. Otherwise out-of-proportion causal claims could still be true, and it would still be true (even if sub-optimal in some sense) to say that 'the purple flame caused the curtains to ignite' in my explicit version of the example. The proportionality-based solution to Explicit Purple Flame requires that we reject that causal claim in favour of citing the flame (purple or otherwise) as the cause of the ignition, but if the out-of-proportion claim remains true, then we have no basis on which to reject it. Only if the strong interpretation of proportionality is true, and so out-of-proportion claims are false in general, can we reasonably rule out the problem links. This would be a significant success and there are those in the literature who do seem to take proportionality to be such a strong constraint: Menzies and List (2010), Sartorio (2010) and Yablo (1992) (though I believe the connection to transitivity is novel).

On the other hand, if proportionality is a constraint on which causal claims are true, then that would rule out many of the canonical claims of causation that we ordinarily endorse: it would be false (and not just infelicitous) to attribute Sophie's peck to the placing of the scarlet triangle, it would be false to say 'the slamming door caused the baby to wake' or to claim that being shot by Mark David Chapman was what caused John Lennon to die. These would be false because there is some more proportional claim: that it was the placing of a red triangle, the making of a loud noise or being shot by someone that did the causal work. We can perhaps grant that such ordinary causal claims are not optimally informative of the causal structure, but surely we do not want to consider them false. If such claims really are false, then much of our ordinary causal talk is literally false. Imposing proportionality as a strong constraint on causation seems like a non-starter if we are to take our ordinary claims seriously. ${ }^{13}$

So, we have a dilemma: if the proportionality constraint is a strong constraint then we can resolve the mismatch problems of transitivity, but at the cost of rejecting much of our ordinary talk as false, but if it is a merely a weak constraint then we can rescue our ordinary causal talk but at the cost of our solution to those problems.

In what follows I will make the case that we should embrace the second horn of the dilemma and that doing so is not as costly as it might appear.

\subsection{Proportionality as a strong constraint}

If we suppose that the proportionality constraint is indeed a strong constraint, then all causes are proportional and so there is no such thing as an out-of-proportion cause. That being so, the following thesis should be exactly equivalent to TRANSITIVITY- C introduced earlier:

\footnotetext{
13 Note that the examples given are clearly assertable, so they tell against even the pragmatic reading of the proportionality constraint discussed in Shapiro and Sober (2012).
} 
PROPORTIONAL TRANSITIVITY- C: If $c_{m}$ is a proportional cause of $d_{p}$ and $d_{p}$ is a proportional cause of $e_{n}$, then $c_{m}$ is a proportional cause of $e_{n}$.

Notice that this thesis is committed to the proportionality of the first cause $\left(c_{m}\right)$ with respect to the eventual effect $\left(e_{n}\right)$ when each step is proportional. So, this thesis makes an important prediction: in every genuine causal chain, the first cause will be proportional with respect the last effect. I think a familiar type of example from the causal literature shows such a prediction to be false: cases of early pre-emption.

In Lewis's original (1973) presentation of his counterfactual analysis of causation, he introduced cases of early pre-emption ${ }^{14}$ to show why he needed causation to be the ancestral of, and not straightforwardly identical with, counterfactual dependence between distinct events. Early pre-emption cases are those where it is intuitively obvious that one event $\left(c_{1}\right)$ caused the effect $(e)$ but where there is no counterfactual dependence of the effect upon that event because there is an unused back-up $\left(c_{2}\right)$ which guaranteed that the effect would occur. Here is a classic example:

\section{Early Pre-emption:}

Billy and Suzy are out to vandalise. Suzy picks up the only rock and throws it towards the window. If Suzy hadn't thrown the rock, Billy would have (and he is notoriously accurate). The rock strikes the window and the window breaks.

Here it is obvious that Suzy is a cause of the window's breaking but it is equally obvious that the breaking did not depend on Suzy because Billy was ready to step in. More precisely, 'if Suzy had not thrown the rock then the window would not have broken' is false because Billy would have broken it in any case. So, there is no overall counterfactual dependence of the window breaking on Suzy's throw. Does this mean that the counterfactual analyst about causation must deny that Suzy is a cause? No, said Lewis. Causation is transitive but counterfactual dependence is not. So whilst there was a failure of overall dependence of the effect upon the initial cause in this case, there was nevertheless a chain of dependence leading from the throw to the window breaking: the rock's being at that point in mid-air (event $d$ ) depended on Suzy throwing it $\left(c_{1}\right)$, and by the time the rock is at that point Billy has been frustrated ( $c_{2}$ had not occurred), and so as of that moment the window's breaking $(e)$ depends upon the rock being at that point $(d)$. Therefore, $e$ depends on $d$, and $d$ depends on $c_{1}$. There may be no overall dependence of $e$ upon $c_{1}$ but there is a step-wise chain of dependence from $c_{1}$ to $e$ via $d$ and so, by the transitivity of causation, $c_{1}$ is a cause of $e$. Thus, by appeal to some version of the transitivity thesis, the counterfactual theorist need not worry about cases of early pre-emption.

However, this neat response does not work if the version of the transitivity thesis appealed to is PROPORTIONAL TRANSITIVITY- C. Notice that Suzy's throwing the rock as she did was proportional to its being at that point in mid air. Simply specifying

\footnotetext{
14 Cases of late, super and trumping varieties of pre-emption came later-see Lewis (1986), Hall (2000) and Schaffer (2000) for discussion. Whilst important cases, they differ in structure from early pre-emption because they are each immune to the transitivity-based response Lewis gives in the original case. Thus, they are not relevant to my discussion here. However, see Bernstein (2014) and McDonnell (2016) for critical discussion of these cases.
} 
'a child's throwing the rock' would be too general, it would imply (wrongly, we can suppose) that Billy's throw would have passed through the same point. However, had Suzy not thrown the rock, and Billy had, the window would have still broken. So Suzy's throw is not a proportional cause of the breaking, a child's throw is. What this means is that whilst the proportional causal chain runs from Suzy's throw to the window breaking, it does not result in a proportional dependence of the window's breaking on Suzy's throw. This contradicts PROPORTIONAL TRANSITIVITY- C. A chain of proportional causation does not necessarily yield proportionality overall.

It is important to note that this line of argument is not based on producing a counterintuitive result, in the manner of Purple Flame and Dog Bark from earlier. Rather, this argument shows that the PROPORTIONAL TRANSITIVITY-C thesis, which is a direct consequence of combining the transitivity of causation with a strong proportionality constraint, is false. The thesis makes a prediction about there being overall proportionality between the first and last steps in a causal chain and that prediction fails in cases with an early pre-emption structure. Thus the thesis is not generally true.

The foregoing should make us question the strategy of combining a transitivity thesis and a strong proportionality constraint on causation. However, the same Early Pre-emption example gives us reason to question the strong constraint directly. Suzy's throwing the rock is not a proportional cause of the window breaking, a child's throw is. So, if adopting the proportionality constraint requires that we reject out-of-proportion causes, then it requires that we deny that Suzy's throwing the rock caused the window to break. Such a result would be at odds with a decades-old consensus in the causal literature: the pre-emptor is the cause. I think this gives us sufficient reason to reject the strong interpretation of the proportionality constraint and with it the first horn of the dilemma.

\subsection{Proportionality as a weak constraint}

Recall that the problem with the initial TRANSITIVITY- C thesis was that it did not restrict its predictions of causal chaining to only proportional causal links. As we have just seen, the problem with PROPORTIONAL TRANSITIVITY-C (which was a consequence of the strong interpretation) is that it wrongly predicts overall proportionality when all the links are proportional. This leaves room for a third alternative:

PROPORTIONAL CHAINING: If $c_{m}$ is a proportional cause of $d_{p}$ and $d_{p}$ is a proportional cause of $e_{n}$, then $c_{m}$ is a cause of $e_{n}$.

The important shift here is that whilst the links in the causal chain must be proportional, the overall causal connection from the first event to the last need not. So, PROPORTIONAL CHAINING does not make the same problematic prediction that PROPORTIONAL TRANSITIVITY-C made in the early pre-emption case, but it still rules out the problematic cases of Purple Flame, Dog Bite and Explicit Purple Flame that were counterexamples to the initial TRANSITIVITY- C (since each have out-of-proportion steps, PROPORTIONAL CHAINING is not committed to the problematic conclusions). This thesis gives us the causal chaining that we want, without the problematic conclusions that we don't in those cases. 
There are two important things to note about PROPORTIONAL CHAINING. First, since this thesis distinguishes causes from proportional causes, it had better be read in light of a weak, rather than strong, interpretation of the proportionality constraint. Otherwise, the final clause in PROPORTIONAL CHAINING is exactly equivalent to the final clause in PROPORTIONAL TRANSITIVITY-C, collapsing the all-important distinction between the two theses. Second, PROPORTIONAL CHAINING is not a transitivity thesis: the relation referred to in the consequent is one about causation, and the relations in the antecedent concern proportional causation. In other words, one can deny the transitivity of causation and still embrace PROPORTIONAL CHAINING.

A question remains, however, about exactly what the proportionality constraint is constraining on this weaker reading. I think this is an interesting question, perhaps an urgent one if my foregoing reasoning is correct, and whilst I have not yet settled on a satisfying answer, I think a recent proposal sketched by Weslake points in the right direction. Weslake sees the role of proportionality as psychological, rather than metaphysical, so it can explain our causal judgements but it cannot constrain the causal truths. On such a reading we need only see the explanatory value in the more proportional causal claims, to understand why we might prefer to assert them in certain cases. This explanatory value is defeasible, however, and to be balanced with other competing values (see Weslake 2010, 2013 for details).

I think that this proposal is promising, but as it stands it will not be able to explain why proportional causal claims successfully form chains, and out of proportion claims do not. My contention here has been that the mismatch cases trade on building too much irrelevant detail into the specification of the events involved, and whilst that irrelevant detail does not make the component causal claims any less true, the special subclass of true causal claims that do not build in such irrelevant detail, are those which successfully form causal chains. The proportionality constraint appears to pick out that subclass.

Yet, even without a complete account of why proportionality licenses chaining, I can grasp the second horn of the dilemma: I deny that causation is transitive in light of the mismatch problems discussed above, but still embrace PROPORTIONAL CHAINING. What we were interested in to begin with was the passing of a causal mark along a causal chain, not transitivity per se. We can retain that important feature without paying the price of full-blown transitivity. I think that this is progress.

\subsection{Switching and short circuits}

At the beginning of the paper, I set aside switching and short circuit examples to focus on mismatch cases. What I have now said about those mismatch cases, about the more difficult sort of case we can create, and about proportionality, will leave those switching and short circuit cases untouched: if they were counterexamples before, they remain so to the revised thesis I offer. That is because the mismatch cases had a different structure all along: they traded on a mismatch in which aspect was relevant at each step. The short-circuit and switching cases, by contrast, have more in common with cases of causal redundancy: the switch, or the short-circuiting mechanism, are parts of a relevant causal chain, just one that makes no difference to the eventual effect. 
I think that this difference in structure justifies a divide and conquer strategy, one which considers the cases separately. This is what I have done here. My conclusions about the mismatch cases leave a range of responses to the switching and shortcircuit cases open: we could accept the counter-intuitive conclusions (as Hall 2000, pp. 205-210 argues we should in switching cases, cf. Sartorio 2005); we could develop treatments within modelling frameworks (see Weslake forthcoming for discussion); or we could bundle these cases together with cases of pre-emption and overdetermination as cases which reveal deep problems with dependence accounts of causation. However we proceed in discussing those cases, the observations about the easy creation of mismatch examples and proportionality that I have discussed here will remain important to bear in mind.

\section{Conclusion}

I have argued here that certain existing putative counterexamples to TRANSITIVITY- C fail to hit their mark as they equivocate on the middle place of the would-be transitive chain. This is a familiar approach to these examples, however I have shown that we can construct a genuine counterexample by making explicit which essence is to be associated with the middle event. ${ }^{15}$ The new counterexample of Explicit Purple Flame is contrived, certainly, but it is a genuine counterexample nevertheless.

The insight offered in response to this new case, and to mismatch counterexamples more generally, is that when they appear to refute the transitivity thesis, they also violate proportionality. That is, at least one of the causal claims that constitute the chain is not proportional in a sense which is related to Yablo's proportionality constraint. Not only does this show us what goes wrong with Explicit Purple Flame, it also explains the inelegance of the example: we prefer proportional explanations. The proposal in this paper is that we consider proportionality to be a constraint on which causal chains entail a causal connection between the first event and the last linked by that chain.

This proposal shines a light on two alternative readings of the proportionality constraint and generates a dilemma: if the proportionality constraint is a strong constraint (i.e. it constrains which claims are true) then we can resolve the mismatch problems of transitivity, but at the cost of rejecting much of our ordinary talk as false, but if it is merely a weak constraint (i.e. it picks out a special subclass of true claims) then we can rescue our ordinary causal talk but at the cost of our solution to the mismatch cases. I have argued that a strong interpretation of the role of proportionality is untenable-it falsifies much of our ordinary causal talk and leads to false conclusions in early pre-emption style cases — but I have also argued that the costs of endorsing a weak interpretation are not as significant as they appear. We can retain the benefits of chaining without incurring the costs of full-blown transitivity by adopting the PROPORTIONAL CHAINING thesis. I conclude that causation is not transitive, but, pending a satisfactory treatment of switching and short-circuit cases, we may not need it to be.

\footnotetext{
15 Here I adopted my preferred counterpart-theoretic approach to events, though I intend nothing of substance to hang on that choice in this discussion. I suspect that the entirety of my case here could be made, albeit less neatly, using an event-aspects theory of the causal relata.
} 
Acknowledgements I am grateful to Umut Baysan, Helen Beebee, Gergely Kertesz, Stephan Leuenberger, Christian Loew, Fiona Macpherson, Peter Menzies, Steph Rennick, Stefan Roski, Nathan Wildman, and Richard Woodward for valuable feedback on earlier drafts. The research for this paper was conducted jointly at the University of Glasgow as part of the Glasgow Emergence Project (funded by John Templeton Foundation Grant 40485) and at Universität Hamburg, as part of the DFG Emmy Noether Research Group Ontology After Quine (WO-1896/1-1). I am thankful for the generous support of the John Templeton Foundation and the Deutsche Forschungsgemeinschaft.

Open Access This article is distributed under the terms of the Creative Commons Attribution 4.0 International License (http://creativecommons.org/licenses/by/4.0/), which permits unrestricted use, distribution, and reproduction in any medium, provided you give appropriate credit to the original author(s) and the source, provide a link to the Creative Commons license, and indicate if changes were made.

\section{References}

Beebee, H., \& MacBride, F. (2014). De re modality, essentialism, and Lewis's Humeanism. In B. Loewer \& J. Schaffer (Eds.), Blackwell companion to David Lewis. New York: Blackwell.

Bernstein, S. (2014). A closer look at trumping. Acta Analytica, 30, 41-57.

Ehring, D. (1987). Causal relata. Synthese, 73(2), 319-328.

Hall, N. (2000). Causation and the price of transitivity. Journal of Philosophy, 97(4), 198-222.

Hitchcock, C. (2001). The intransitivity of causation revealed in equations and graphs. The Journal of Philosophy, 98(6), 273-299.

Lewis, D. (1968). Counterpart theory and quantified modal logic. Journal of Philosophy, 65(5), 113-126.

Lewis, D. (1973). Causation. Journal of Philosophy, 70(17), 556-567.

Lewis, D. (1986). Postscripts to causation. In D. Lewis (Ed.), Philosophical papers (Vol. II, pp. 172-213). Oxford: Oxford University Press.

Lewis, D. (2001). Counterfactuals. Oxford: Blackwell.

Lewis, D. (2003). Things qua truthmakers. In H. Lillehammer \& G. Rodriguez-Pereyra (Eds.), Real metaphysics: Essays in honor of D. H. Mellor (pp. 25-38). London: Routledge.

Lewis, D. (2004). Causation as influence. In J. Collins, N. Hall, \& L. A. Paul (Eds.), Causation and counterfactuals (pp. 75-106). Cambridge, MA: The MIT Press.

Mackie, J. L. (1980). The transitivity of counterfactuals and causation. Analysis, 40(1), 53-54.

McDermott, M. (1995). Redundant causation. British Journal for the Philosophy of Science, 46(4), 523-544.

McDonnell, N. (2016). Events and their counterparts. Philosophical Studies, 173(5), 1291-1308. doi:10. 1007/s11098-015-0547-5.

Menzies, P. (2014). Counterfactual theories of causation. In E. N. Zalta (Ed.), The Stanford encyclopedia of philosophy, spring 2014 edition. Stanford: The Metaphysics Research Lab.

Menzies, P., \& List, C. (2010). The causal autonomy of the special sciences. In C. McDonald \& G. McDonald (Eds.), Emergence in mind. Oxford: Oxford University Press.

Paul, L. A. (2000). Aspect causation. Journal of Philosophy, 97(4), 235-256.

Sartorio, C. (2005). Causes as difference-makers. Philosophical Studies, 123(1-2), 71-96.

Sartorio, C. (2010). The prince of wales problem for counterfactual theories of causation. In A. Hazlett (Ed.), New waves in metaphysics (pp. 259-276). New York: Palgrave McMillan.

Schaffer, J. (2000). Trumping preemption. Journal of Philosophy, 97(4), 165-181.

Schaffer, J. (2005). Contrastive causation. Philosophical Review, 114(3), 327-358.

Shapiro, L., \& Sober, E. (2012). Against proportionality. Analysis, 72(1), 89-93.

Weslake, B. (2010). Explanatory depth. Philosophy of Science, 77(2), 273-294.

Weslake, B. (2013). Proportionality, contrast and explanation. Australasian Journal of Philosophy, 91(4), $785-797$.

Weslake, B. (forthcoming). A partial theory of actual causation. British Journal for the Philosophy of Science.

Yablo, S. (1992). Mental causation. Philosophical Review, 101(2), 245-280. 